\title{
Modelling of Blood and Dialysate Flow in Hollow-Fiber Hemodialyzers
}

Hamid $\mathrm{H}^{*}$, Mohammad JA and Sobhani FK

Department of Petroleum and Chemical Engineering, Sharif University of Technology, Tehran, Iran

\begin{abstract}
One of the problems that mostly arises in kidney diseases, is abnormal reduction of fluid removal in the body, which increases the level of acids and increase normal range of molecules such as urea, glucose, creatinine, beta 2 -micro globulin and complement factor D. Due to the availability of technologies to produce membranes and fibers, it is possible to remove these toxins by producing membrane sets, such as dialysis. The heart of a dialysis is dialyzer that filters toxins and extra water of the body through its hollow membranes. In this regard, a model is needed to investigate and optimize process of toxin clearance, in order to reduce manufacturing costs and increase efficiency of dialysis. Since process parameters and dialysis structure determines elimination level and also the stay of useful molecules in blood, manufacturing design is of great importance. In this paper, a two-dimensional model of speed, pressure and flow concentration was prepared for both blood and dialysate phase, and discretized using finite difference method. Here, the simulation was carried out using MATLAB software. TIn he present work we try to build a 2-D model of blood and dialysate flows as a platform to study dialyzer performance in the future.
\end{abstract}

Keywords: Dialysis; Simulation; Modelling; Membrane fibers

\section{Introduction}

Hemodialysis is essentially a filter for blood flow, which cleans blood toxins of patients with artificial kidney. This blood filtering system is capable to be mounted on a patients body in hospitals and even at home, while it doesnt need special care during the filtering [1]. In fact, the process of dialysis plays the role of a kidney and is of use for kidney patients. During hemodialysis process, the patient's blood drives to very thin porous fibers [2]. In the case of homogeneous membranes for dialysis, the literature witnesses considerable and valuable researches. Such dialysis membranes are generally made using cellulosic materials, in which a homogeneous porous structure is present from the membrane entry to the exit. The surface of the membranes are not very biocompatible with blood flow because of the exposure in the hydroxyl groups. That is why dialysis membranes are generally made of synthesized materials such as poly aryl, ether sulfate, polyimide, polycarbonate and polyacrylamide. This type of membrane, known as asymmetric structure, is less active compared to cellulose membranes, which means that its hole shapes gradually change from the entry to the exit of membrane surface. Although synthesized membranes are mostly in the form of high flux, they can be made in both high and low flux forms [3]. The majority of hemodialysis membranes are designed for toxic clearance and prevention of protein penetration, and thus, the design should prevent back filtration [4]. Although, experimental studies conducted so far have investigated what is the effect of blood or dialysate flow rate on the elimination of toxins [5], they have not examined membrane structure, and also have usually been limited to the removal of urea. One of the main challenges ahead of clinicians is the duration of dialysis procedures in case of penetration of important molecules, such as albumin material. Most laboratory studies about dialysis are commercially available and in fact provide a comparison between different dialyzers [4]. In a dialysis, materials, including sodium, potassium, calcium, magnesium, chloride, bicarbonate and glucose, move to dialysate solution. In the meantime, toxins and excess water will be removed from blood. For maximization of theefficiency, the blood and dialysate flow will be counter-current. This process continues until the concentration of undesirable materials reaches to normal-level. The size of the pores should be small enough to prevent transmission of blood cells, but pass undesirable materials. The purification process usually takes three to five hours and is done two or three times a week
[2]. During the operation, blood distributes in the fibers, and excess water and toxins move into the dialysis solution due to pressure and concentration difference with the external environment of the fiber. Figure 1 shows how the dialyzer device installs on the body's patients. Schematics of blood current outside the patient's body by hemodialysis [1] (Figure 1). One of the efforts in this regard is simulating how mass transfers in a porous fiber [6]. In the present model, angle gradient terms are ignored in blood and dialysate flows. paper.

\section{Governing Equations}

This paper is focused on the simulation of counter-current blood and dialysate solution flows. The transfer of the afore mentioned materials in membrane shell is similar to heat transfer in shell and tube converters [3]. In the present model, it is assumed that laminar flow conditions are perfectly preserved. To investigate different structural and operational parameters, the authors studied filter F60 (Fresenius, Gemrany), that its structural and morphological characteristics are available in references [1].

\section{Differential equations of concentration and velocity of blood flow}

In dialysis input flow due to current contact with the membrane's inner walls, the velocity distribution will be developed that is considered as an input boundary condition for velocity.

$$
U_{z}(\mathrm{r})=\frac{2 q b}{N_{b}^{4}}\left(r_{b}^{2}-r^{2}\right) \text { at } z=0
$$

*Corresponding author: Hamid H, Department of Petroleum and Chemica Engineering, Sharif University of Technology, Tehran, Iran, Tel: 982166165421 ; E-mail: Hamidhamed1990@gmail.com

Received: December 11, 2018; Accepted: January 30, 2018; Published: February 10, 2018

Citation: Hamid H, Mohammad JA, Sobhani FK (2018) Modelling of Blood and Dialysate Flow in Hollow-Fiber Hemodialyzers. J Bioengineer \& Biomedical Sci 8: 242. doi: 10.4172/2155-9538.1000242

Copyright: $\odot 2018$ Hamid H, et al. This is an open-access article distributed under the terms of the Creative Commons Attribution License, which permits unrestricted use, distribution, and reproduction in any medium, provided the original author and source are credited. 


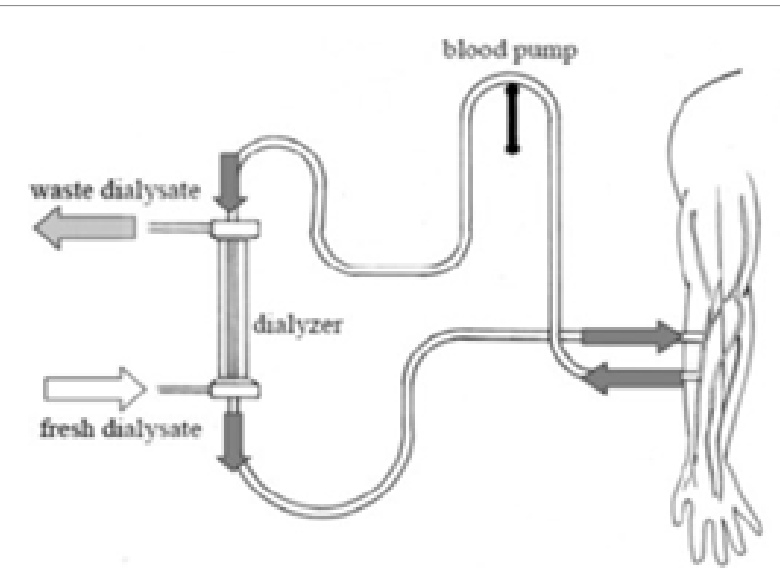

Figure 1: Schematics of blood current outside the patient's body by hemodialysis.

Eq. 1 provides the velocity distribution of blood at the entrance of a fiber. Here, $\mathrm{qb}$ is the input flow rate of blood, which determines a velocity distribution from zero at radius rb to fiber axis. It is assumed that there are no sliding conditions in the wall and there is fiber symmetry axis at $r=0$ [7]. At totally developed flow conditions, Navier Stokes equations and continuity equation are governed in accordance with the following process, by the assumption that the dialysate and blood flow are Newtonian and incompressible [7].

Here the parameter $\mathrm{q}_{d}$ indicates the flow rate at the dialysate entrance and the ratio $k\left(\left(r_{b} / r_{d}\right)\right)$ and $L_{r}$ are the structural jbcharacterisitics of the hollow fiber [7].

$$
\begin{aligned}
& \frac{1}{r} \frac{\partial(\mathrm{rUr})}{\partial r}+\frac{\partial U_{Z}}{Z}=0 \\
& \mathrm{Ur} \frac{\partial(\mathrm{Ur})}{\partial r}+U_{Z} \frac{\partial U_{r}}{\partial z}=-\frac{1}{\rho}+\frac{\mu}{\rho}\left[\frac{1}{r} \frac{\partial\left(r \partial U_{\mathrm{r}}\right)}{r}-\frac{U_{\mathrm{r}}}{r^{2}}+\frac{\partial^{2} U r}{\partial z^{2}}\right] \\
& \mathrm{Ur} \frac{\partial(\mathrm{Uz})}{\partial r}+U_{Z} \frac{\partial U_{z}}{\partial z}=-\frac{1}{\rho}+\frac{\mu}{\rho}\left[\frac{1}{r} \frac{\partial\left(r \partial U_{z}\right)}{r}+\frac{\partial^{2} U z}{\partial z^{2}}\right]
\end{aligned}
$$

Here, $\mathrm{u}_{\mathrm{r}}$ and $\mathrm{u}_{\mathrm{z}}$ are radial and axial velocities respectively, and $\mathrm{p}$ is operation pressure. Continuity relation is used for the balance of the number of the equations and the unknowns, because of the consideration of the pressure distribution of flow. Convection-diffusion equation governing mass transfer of solutes can be seen below, that will be coupled with velocity distribution field.

$$
\mathrm{Uz} \frac{\partial C s}{\partial z}+\mathrm{Ur} \frac{\partial C s}{\partial r}=D_{e f f}\left[\frac{\partial^{2} C s}{\partial r^{2}}+\frac{1}{r} \frac{\partial C s}{\partial r}+\frac{\partial^{2} C s}{\partial z^{2}}\right]+R s
$$

In above equation, $\mathrm{C}_{\mathrm{s}}$ and $\mathrm{D}_{\mathrm{s}}$ are concentration and effective diffusion coefficient for both blood and dialysate phase, while $\mathrm{R}_{\mathrm{s}}$ takes a value of zero as a source of production or consumption in the present work. The bracketed terms are related to diffusion, while the second and third terms on the left side are related to the convection of solutes by the flow [7]. Effective diffusion coefficient depends strongly on the morphological properties of the hollow-fiber. This can be defined as [8]:

$$
D_{e f f}=D f(\mathrm{q}) \mathrm{S}_{\mathrm{D} A k}
$$

$$
\begin{gathered}
q=\frac{r_{s}}{r_{p}} \\
f(\mathrm{q})=\frac{1-2.1050 q+2.0865 q^{3}-1.7068 q^{5}+0.72603 q^{6}}{1-0.75857 q^{5}} \\
\mathrm{~S}_{\mathrm{D}}=(1-q)^{2}
\end{gathered}
$$

In these equations, $\mathrm{D}$ reffers to the diffusion coefficient of solutes in the same solutions and a non-porous medium and is a function of solute's molecular weight. $r_{s}$ and $r_{p}$ also represent the solute and pore radius respectively [8].

The velocity distribution of the input flow in the dialysate solution is considered accordance with equation (9).

$$
\mathrm{U}_{\mathrm{z}}=\frac{2 q d}{\pi r_{d} L_{r}}
$$

Here the parameter qd indicates the flow rate at the dialysate entrance and the ratio $\mathrm{k}((\mathrm{rb} / \mathrm{rd}))$ and $\mathrm{Lr}$ are the structural characterisitics of the hollow fiber [7].

\section{Boundary conditions}

In this work, initial and boundary conditions determine the velocity and concentration distribution of the profile. The following are mentioned as the initial and boundary conditions that in papers are totally common. Due to the symmetry of fiber, let: [7]

$$
\begin{aligned}
& \frac{\mathrm{d} U_{\mathrm{z}}}{d r}=0 \text { at } r=0 \\
& \frac{d U_{r}}{d r}=0 \text { at } r=0
\end{aligned}
$$

Velocity at the surface of fiber membrane is assumed to be zero at axis. Accordingly, we have:

$$
\mathrm{U}_{\mathrm{z}}=0 \text { at } r=R_{\text {in }}
$$

Also, at the fiber axis of $r=0$, radial velocity would be considered equal to zero for both blood and dialysis phase. In membrane surface, due to the flow of water and materials to the dialysis solution, the following equation holds for radial velocity:

$$
U_{r}=J_{v} \text { at } r=R_{\text {in }}
$$

Here $J_{v}$ represents the ultrafiltration flux and depends on the pressure differences between blood side and dialysate side. Hydraulic permeability relates these two values and is one of the most important physicochemical properties of the hollow-fibers:

$$
J_{v}=L_{p}\left[P_{B}-P_{D}\right]
$$

For the output boundry, output pressure is considered as a boundary condition (equation (12)) and it can be used as a key parameter in determining the level of flux of blood to the dialysis solution, or vice versa.

$$
\mathrm{P}=\mathrm{P}_{0} \quad \text { at } z=0
$$

Influx and outflux boundary conditions are in accordance with the equation (15) [7] for the concentration equation.

$$
C_{s}(z, r, 0)=C_{s 0}, C_{s}(0, r, t)=C_{s 0}, \frac{\partial C_{s}(\mathrm{z}, 0, \mathrm{t})}{\partial r}=0
$$




\section{Numerical Solution}

In this paper, the numerical solution was provided using finite difference method. All differential equations were discrete in the form of a finite difference. For a discrete of the first and second order derivatives, central difference estimate was used in accordance with the following relationships.

$$
\begin{aligned}
& \frac{\partial^{2} U}{\partial x^{2}}=\frac{U_{i+1, j}^{n+1}-2 U_{i, j}^{n+1}+U_{i-1, j}^{n+1}}{\Delta x^{2}}, \frac{\partial^{2} U}{\partial y^{2}}=\frac{U_{i, j+1}^{n+1}-2 U_{i, j}^{n+1}+U_{i-1, j-1}^{n+1}}{\Delta x^{2}} \\
& \frac{\partial^{2} C_{s}}{\partial x^{2}}=\frac{C_{S_{i+1, j}}^{n+1}-2 C_{S_{i, j}}^{n+1}+C_{S_{i-1}, j}^{n+1}}{\Delta x^{2}}, \frac{\partial^{2} C_{s}}{\partial y^{2}}=\frac{C_{S_{i, j}}^{n+1}-2 C_{S_{i, j}}^{n+1}+C_{S_{i, j-1}}^{n+1}}{\Delta y^{2}} \\
& \frac{\partial C_{s}}{\partial x}=\frac{C_{S_{i+1, j}}^{n+1}-C_{S_{i-1, j}}^{n+1}, \frac{\partial C_{s}}{\partial y}=\frac{C_{S_{i, j}}^{n+1}-C_{S_{i, j-1}}^{n+1}}{2 \Delta y}}{2 \Delta x}
\end{aligned}
$$

In order to discretize the time derivative, the following formulae were utilized.

$$
\frac{\partial C_{s}}{\partial t}=\frac{C_{S_{i, j}}^{n+1}-C_{S_{i, j}}^{n}}{\delta t}
$$

\section{Results and Discussion}

Due to the boundary conditions, it is clear that the maximum velocity will be at the axis of fiber, while it decreases to zero on the surface of the membrane's wall. It should be noted that various parameters affect velocity distribution, including level of the flux in and out of the membrane and solution pressure (Figure 2).

From Figure 3, it could be seen that the total flux of blood out of the membrane was in some parts positive and in some places negative. The parameter that determines the positive and negative in and out flux

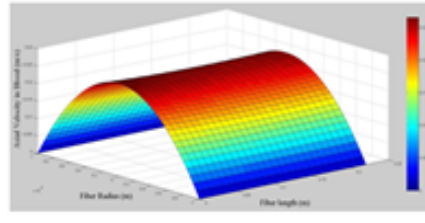

(a)

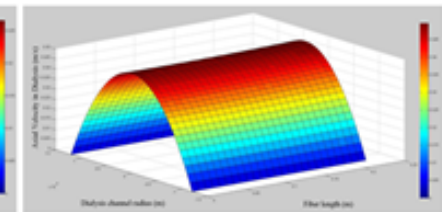

(b)
Figure 2: Distribution of axial velocity for both blood (a) and dialysate phase (b).

Idealized geometry

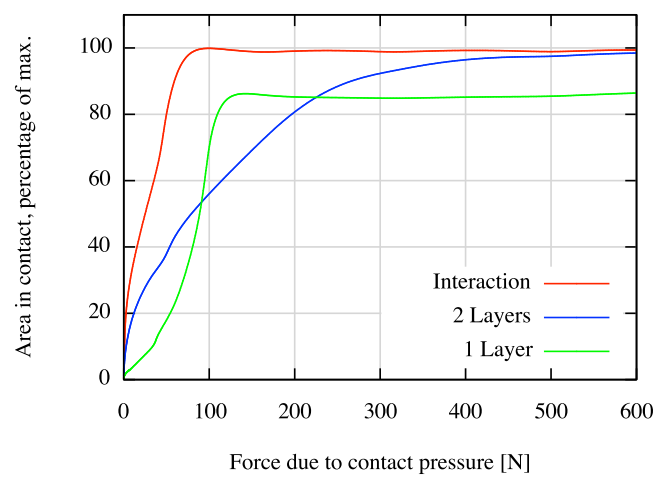

Figure 3: Radial velocity for two phases: blood (a) and dialysate (b). (a)

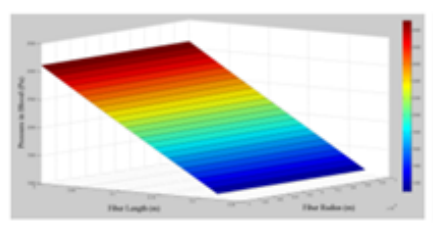

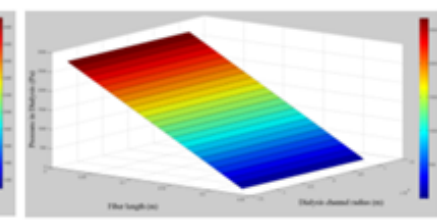

(b)
Figure 4: Pressure for both phase of blood (a) and dialysate (b).

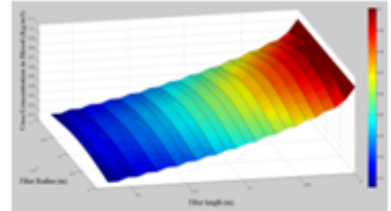

(a)

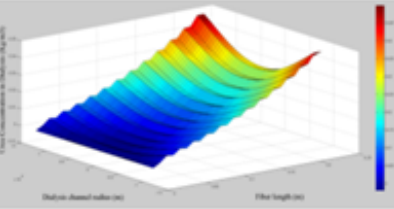

(b)
Figure 5: Distribution of urea concentration in blood (a) and dialysate (b) compartments.

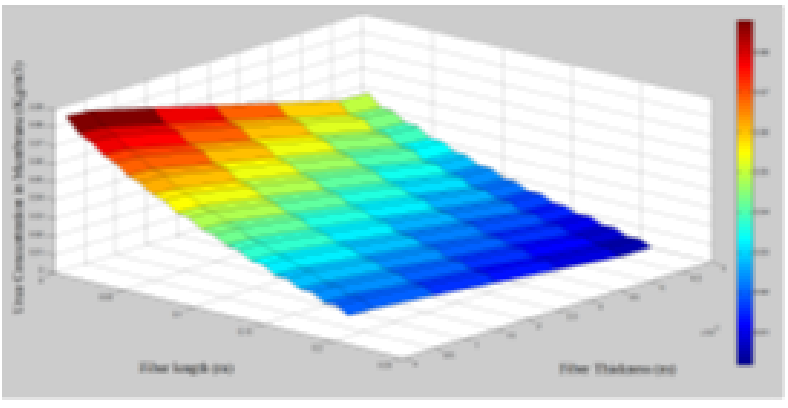

Figure 6: Distribution of urea concentration within membrane layer.

(mainly water and dialysate solution) is membrane input and output pressure for both blood and dialysate phase. Also by careful observation at shells, it is characterized that in the maximum and minimum of the radial velocity was achieved at certain radial points.

According to Figure 4, pressure distribution is descending along the flow direction. Based on the counter currents, the maximum pressure of the dialysis (the input of the dialysate solution) was associated with the lowest pressure in blood output, and vice versa. As it was explained, this pressure difference leads to fluid flow force.

Figure 5 shows how the urea concentration changes with axial and radial dimensions in both blood and dialysate compartments. The concentration profiles show non-linear change of urea concentration along both axial and radial dimensions. Figure 6 shows how urea concentration varies within he membrane thickness. Figure 6 shows how urea concentration varies within he membrane thickness.

\section{Conclusion}

The model developed in this paper can be used to predict solute concentration, pressure and velocity profiles as a function of time and dialyzer structural dimensions. Model predictions have shown that solute concentration in the radial direction which is normally assumed to be uniform in other studies, change nonlinearly. In addition, existence of radial velocity plays an important role in convection transport of larger molecules.

\section{Conflict of Interest}

The authors declare that they have no conflict of interest. 
Citation: Hamid H, Mohammad JA, Sobhani FK (2018) Modelling of Blood and Dialysate Flow in Hollow-Fiber Hemodialyzers. J Bioengineer \& Biomedical Sci 8: 242. doi: 10.4172/2155-9538.1000242

\section{References}

1. Abaci HE, Altinkaya SA (2010) Modeling of hemodialysis operation. Ann Biomed Eng 38: 3347-3362.

2. Haas K (2010) Modeling blood cell concentration in a dialysis cartridge. Ph.D. thesis, Worcester Polytechnic Institute.

3. Islam MS, Szpunar J (2013) Study of dialyzer membrane (polyflux 210h) and effects of different parameters on dialysis performance. O J Neph 3: 161-167.

4. Hedayat A, Szpunar J, Kumar N, Peace R, Elmoselhi H, et al. (2012) Morphological characterization of the polyflux $210 \mathrm{~h}$ hemodialysis filter pores. Int J Nephrol 2012: 304135.
5. Borzou S, Gholyaf M, Zandiha M, Amini R, Goodarzi M, et al. (2009) The effect of increasing blood flow rate on dialysis adequacy in hemodialysis patients. Saudi J Kidney Dis Transpl 20: 639-642.

6. Ding W, He L, Zhao G, Zhang H, Shu Z (2004) Double porous media model for mass transfer of hemodialyzers. Int J Heat Mass Transfer 47: 4849-4855.

7. Annan K (2012) Mathematical modeling for hollow fiber dialyzer: Blood and $\mathrm{HCO}_{3}$ - dialysate flow characteristics. International Journal of Pure and Applied Mathematics 79: 425-452.

8. Preston BN, Comper WD, Hughes AE, Snook I, van Megen W (1982) Diffusion of dextran at intermediate concentrations. J Chem Soc Faraday 78: 1209-1221. 